\title{
Abcès cérébraux d'origine dentaire : à propos de deux cas
}

\section{RÉSUMÉ}

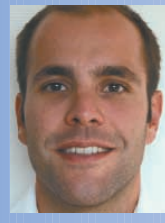

Julien BALLY

Assistant hospitalier universitaire,

Service de chirurgie buccale,

CHU Nancy,

13 , rue Blaise Pascal,

54320 Maxeville.

\section{Rémi CURIEN}

Praticien hospitalier,

CHU Nancy.

\section{Sophie BRENOT}

Chirurgien-dentiste

Nancy.

\section{Alexandra SOURDOT}

Assistant hospitalier universitaire,

Service de chirurgie buccale,

CHU Nancy.

\section{Audrey LÊ}

Assistant hospitalier universitaire, Service de chirurgie buccale,

CHU Nancy.

\section{Pierre BRAVETTI}

Maître de conférence,

Praticien hospitalier

à la faculté d'odontologie de Nancy,

Doyen de la faculté d'odontologie,

Responsable du département

de chirurgie et pathologie buccale,

Service d'odontologie,

CHU de Nancy.

Même si le pronostic des abcès du cerveau s'est amélioré grâce à I'antibiothérapie, l'imagerie et la chirurgie, leurs complications peuvent encore remettre en cause le pronostic vital. Leurs étiologies sont diverses ; nous rapportons ici deux cas d'abcès du cerveau d'origine dentaire. À la lumière de ces cas, il apparaît essentiel comme pour toute infection focale d'éradiquer l'ensemble des foyers infectieux en bouche.

infection dentaire focale

- abcès cérébral
AOS 2009;248:361-367

DOI: 10.1051/aos/2009410

(C) AEOS / EDP Sciences 


\section{Introduction}

Les abcès cérébraux correspondent à des suppurations focales d'origine infectieuse développées au sein du parenchyme cérébral. L'abcès cérébral est une pathologie de pronostic relativement grave. Bien que peu fréquent chez l'immunocompétent, il doit être évoqué et diagnostiqué rapidement pour une prise en charge thérapeutique efficace.

Depuis l'avènement des antibiotiques, l'abcès du cerveau est devenu une pathologie rare. L'infection est dite opportuniste et son incidence est de 2,6 pour 1000 hospitalisations [1]. L'âge moyen est de 30-45 ans [1]. On retrouve $66 \%$ de prévalence masculine [1]. L'incidence générale des abcès cérébraux est d'environ 1/100 000 [2]. Brewer [3] a répertorié, sur 60 cas étudiés, 4 cas d'origine dentaire soit $6,6 \%$. La mortalité suite à un abcès cérébral est estimée à $10 \%$ [4].

Au cours de son développement, l'abcès cérébral à pyogènes passe par deux stades évolutifs principaux : un stade de cérébrite (ou d'encéphalite présuppurative) puis un stade d'encapsulation, à partir du $10^{\mathrm{e}}$ jour, créant une compression des structures adjacentes.

Ils peuvent avoir, en dehors d'un contexte neurochirurgical, deux origines possibles :

- soit une propagation d'un germe par contiguïté provenant d'un foyer septique de voisinage (50\% des cas). Les germes diffusent au travers du système de drainage veineux, en raison de l'existence de communications par des veines perforantes entre les réseaux intra- et exo-crâniens [5]. Le foyer infectieux primitif le plus souvent retrouvé est une sinusite frontale mais il peut également s'agir d'une sinusite ethmoïdale, sphénoïdale ou maxillaire, d'une otite moyenne aiguë ou chronique cholestéomateuse ou encore de foyers infectieux dentaires ou faciaux ;

- soit une dissémination hématogène (25\% des cas), à partir d'un foyer à distance. II s'agit alors plus fréquemment de lésions multiples, avec une capsule moins nette. Le site de départ peut être une endocardite voire un abcès pulmonaire ou abdominal. L'existence d'une cardiopathie congénitale (CIA, CIV ou tétralogie de Fallot) ou d'une maladie de Rendu-Osler avec fistules artérioveineuses est un facteur de risque de développer un abcès cérébral [6]. Pour l'abcès cérébral d'origine hématogène, différentes études montrent une fréquence accrue dans les territoires frontaux, pariétaux ou fronto-pariétaux, souvent associée à une résistance accrue aux antibiotiques [7].

Il reste que dans $25 \%$ des cas aucune étiologie n'est retrouvée.

Au niveau bactériologique, les germes le plus souvent responsables sont les streptocoques et particulièrement ceux du groupe intermedius, dont Streptococcus anginosus [8]. Les abcès cérébraux peuvent être polymicrobiens (40\% des cas), surtout si les germes proviennent de l'oropharynx (association de streptocoques et d'anaérobies). D'autres bactéries peuvent également être retrouvées, en fonction de la porte d'entrée : entérobactéries, pyocyaniques, staphylocoques dorés.

La survenue d'abcès cérébral est largement conditionnée par des facteurs de risque locaux ou généraux. On notera une fréquence plus élevée chez les patients avec des antécédents d'infarctus du myocarde, de cardiopathie congénitale, ceux avec un risque d'endocardite 
infectieuse, les patients immunodéprimés [9] (syndrome d'immunodéficience acquise SIDA, population toxicomane).

L'antibiothérapie de choix, en l'absence d'orientation bactériologique particulière, est I'association d'amoxicilline ou de céfotaxime, aux posologies de 150 à $200 \mathrm{mg} / \mathrm{kg} / \mathrm{j}$ avec le métronidazole à la posologie de $30 \mathrm{mg} / \mathrm{kg} / \mathrm{j}$ [8]. Le traitement antibiotique dans les premières étapes de développement de l'abcès est suffisant avec un contrôle par scanner ou IRM cérébrale de manière à juger de l'évolution favorable.

En cas d'abcès constitué, I'antibiothérapie seule n'est prescrite qu'en l'absence de signes de gravité clinique ou scanographique. La ponction-aspiration de l'abcès en stéréotaxie permet d'obtenir une documentation bactériologique dans plus de $85 \%$ des cas où elle est réalisée [8]. L'indication est donc formelle en cas de tableau neurologique grave, d'abcès volumineux, d'effet de masse important ou de menace de rupture par une localisation au voisinage des ventricules. II reste exceptionnel de devoir recourir à une craniotomie avec excision-drainage. La ponction neurochirurgicale concerne au mieux les abcès de plus de $3 \mathrm{~cm}$, siégeant dans des sites accessibles, sans nécessiter de traverser une région anatomique vitale. La persistance d'images séquellaires au scanner cérébral est habituelle, même à distance de l'arrêt des antibiotiques, alors que l'infection est guérie. Ces séquelles peuvent être la source d'une maladie épileptique résiduelle rendant nécessaire la poursuite d'un traitement anticomitial.

Nous présentons ici deux abcès cérébraux d'origine dentaire probable.

\section{Premier cas clinique}

Un patient âgé de 54 ans est hospitalisé suite à des céphalées, un état confusionnel, des troubles du comportement et de la vigilance.

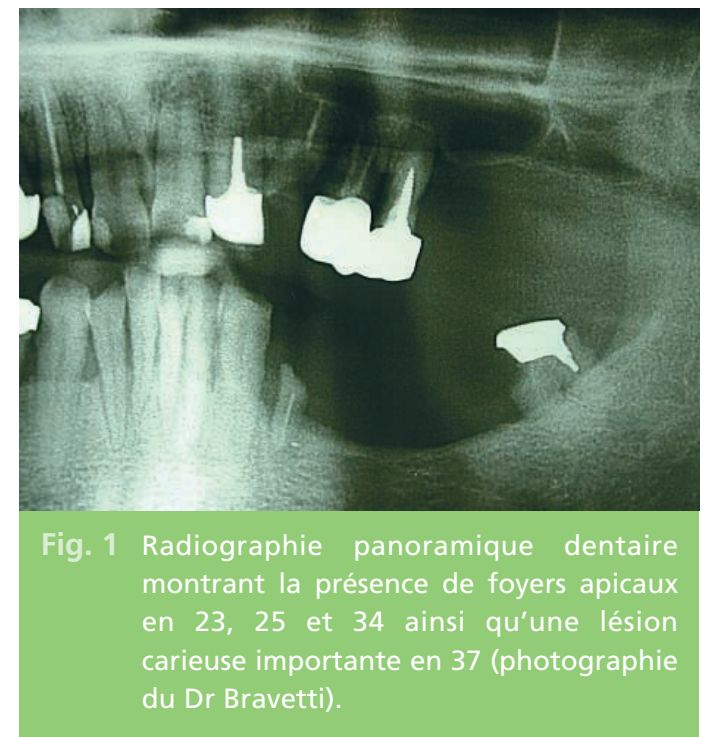

L'examen clinique révèle de plus une aphasie avec signes neurologiques (aréflexie des membres inférieurs et de raideur de la nuque).

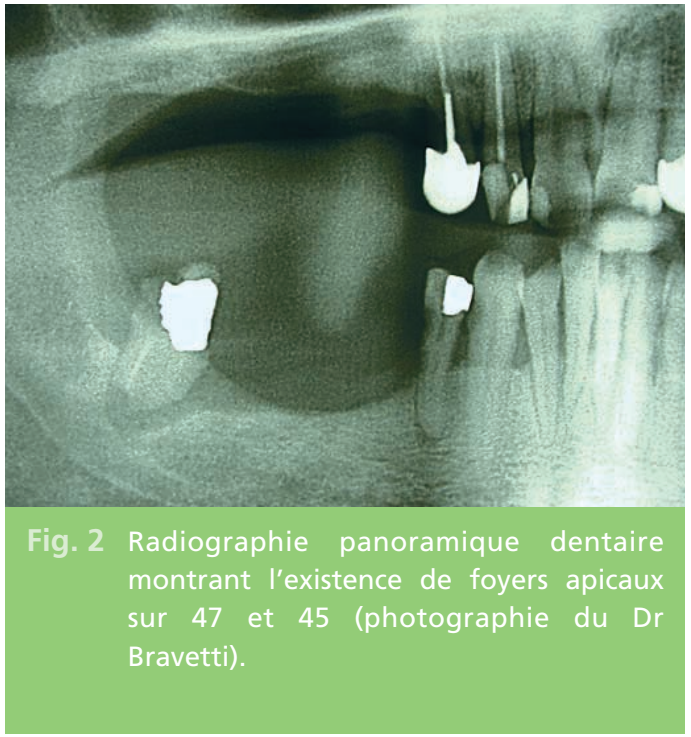


L'examen tomodensitométrique réalisé oriente le diagnostic en révélant la présence de plusieurs foyers hypodenses dans la région frontotemporale, qui conclut à l'abcès cérébral. Le drainage chirurgical n'est pas retenu, la suppuration intracrânienne étant peu étendue. La ponction stéréotaxique qui permet l'aspiration du pus retrouve des germes anaérobies. Un germe est identifié : streptocoque du groupe intermedius. l'étiologie dentaire est privilégiée, aucune autre porte d'entrée n'ayant été retrouvée.
Une antibiothérapie à large spectre (amoxicilline et métronidazole) est mise en place, entraînant la régression totale des symptômes.

Le patient est alors adressé au service de chirurgie buccale. L'examen clinique et radiographique (panoramique dentaire, fig. 1 et 2) montre plusieurs foyers infectieux potentiels avec des lésions apicales et une maladie parodontale. Une remise en état de la cavité buccale est entreprise de manière à éviter toute récidive.

\section{Deuxième cas clinique}

Un patient âgé de 50 ans, sans antécédent ni traitement médicamenteux, est atteint d'une aphasie fébrile qui s'aggrave d'un coma, il est hospitalisé au service de maladie infectieuse pour suspicion d'abcès cérébral.

La tomodensitométrie étant réalisée dès I'admission du patient, cela permet de poser le diagnostic d'abcès du cerveau et d'orienter le geste chirurgical. La tomodensitométrie en

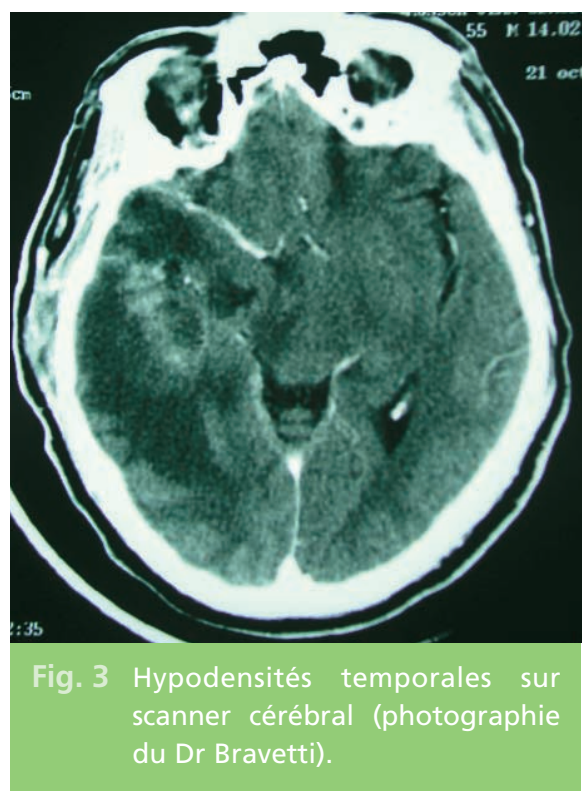

coupe axiale montre dans le plan fronto-pariétal (fig. 3 et 4) un foyer hypodense mesurant $8 \mathrm{~cm}$ d'axe antéropostérieur et $5,5 \mathrm{~cm}$ d'axe transversal. La réalisation de fenêtre osseuse est nécessaire dans ce cas, étant le cadre de foyers infectieux. Le drainage chirurgical effectué, la ponction stéréotaxique montre un germe anaérobie d'origine dentaire : Fusobacterium nucleatum. À l'issue du drainage chi-

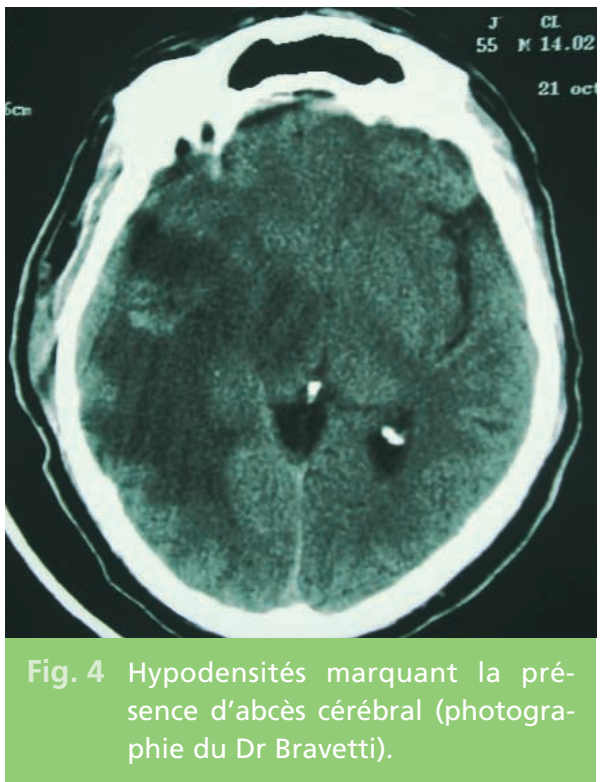




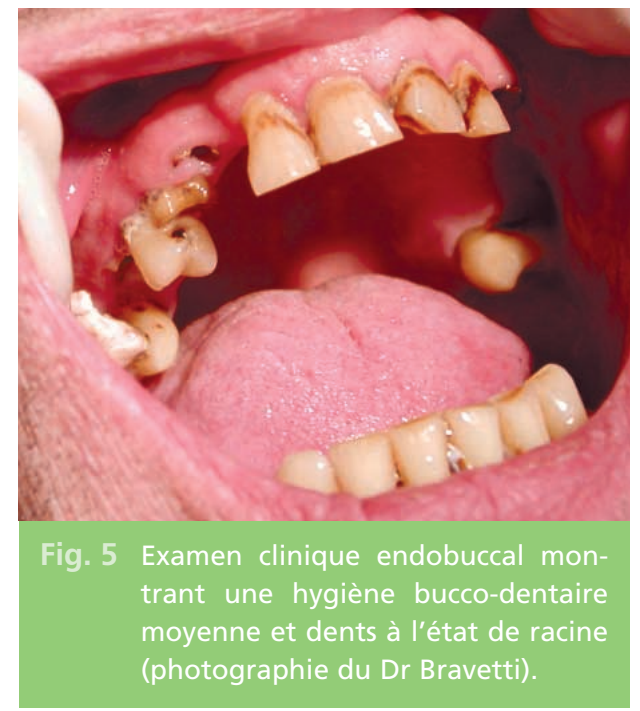

rurgical, une disparition complète et rapide de l'aphasie est observée. Dans le même temps, le patient est placé sous antibiothérapie à large spectre métronidazole et amoxicilline.

Le bilan étiologique n'a retrouvé que la porte d'entrée dentaire compatible avec les examens bactériologiques.

Le patient est donc adressé au service de chirurgie buccale. L'examen clinique et l'examen

\section{Discussion}

On retrouve dans la littérature de nombreux articles montrant les conséquences parfois graves de foyers infectieux dentaires. La diffusion des germes bucco-dentaires au niveau cérébral peut se faire soit par contiguïté dans le cadre d'infection aiguë ou alors par voie hématogène avec les foyers chroniques [10]. La flore microbienne retrouvée dans les abcès cérébraux d'origine dentaire est polymorphe. Dans $70 \%$ des cas, on retrouve les streptocoques [11]. Dans le premier cas, le germe identifié est le streptocoque intermedius, bactérie aérobie-anaérobie facultatif. Cette

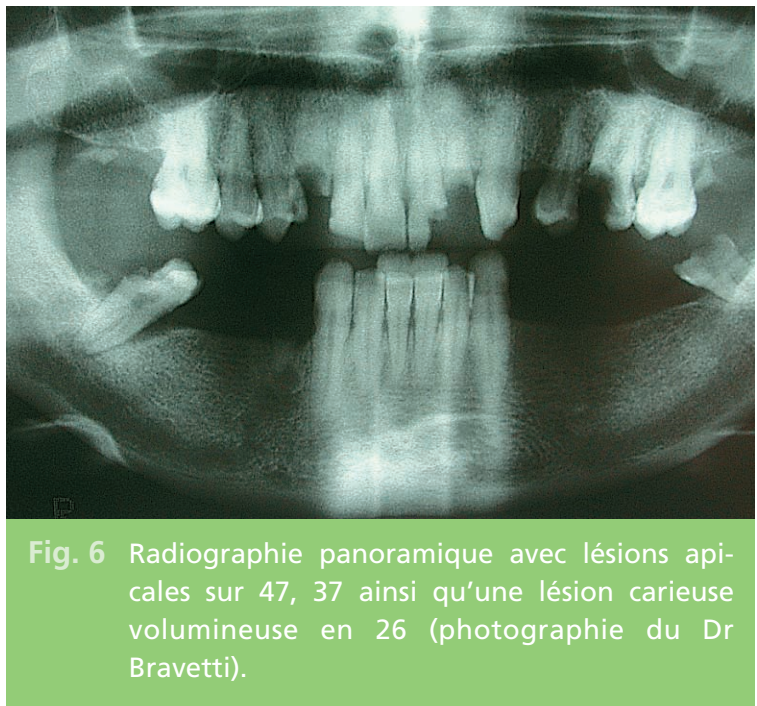

radiographique (fig. 5 et 6 ) montrent une hygiène bucco-dentaire défectueuse ainsi que de nombreux foyers infectieux: alvéolyse horizontale généralisée, plusieurs dents à l'état de racine avec des foyers infectieux apicaux.

Les foyers infectieux sont tous supprimés de manière à éviter tout risque de récidive.

espèce prolifère dans les maladies parodontales et constitue l'espèce principale d'origine buccale retrouvée dans les bactériémies [4]. Fusobacterium qui a été identifié comme responsable dans le deuxième cas clinique correspond à des bâtonnets Gram négatifs qui sont eux aussi fréquemment retrouvés dans les infections à distance. Il est difficile dans les cas présentés d'établir de manière sûre la relation entre les foyers bucco-dentaires et les abcès cérébraux. En effet, devant l'urgence de la situation clinique, la prise en charge médicale vise dans un premier temps à faire régresser 
l'infection. La recherche d'une porte d'entrée n'est effectuée qu'après le bilan bactériologique, le plus souvent avec une antibiothérapie déjà débutée.

L'attitude à adopter par l'odontologiste va être conditionnée par l'état général du patient et une éventuelle immunodépression. Cependant l'éradication des foyers infectieux importants doit être une priorité. Il apparaît difficile de déterminer une classe de patient dit à risque comme on peut le faire pour l'endocardite d'Osler. En effet, I'incidence des abcès cérébraux n'est pas plus importante chez les patients ayant des antécédents de pathologie cérébrale, encéphalites, méningites ou de traumatisme que dans la population générale.

Ces cas démontrent, une fois n'est pas coutume, la possibilité de complications graves découlant de foyers infectieux bucco-dentaires. Comme dans de nombreuses situations d'infections focales d'étiologie dentaire, le foyer infectieux d'origine ne peut être identifié avec certitude mais sur un faisceau d'arguments cliniques et bactériologiques. Toutefois dans chacun des cas où une origine dentaire est suspectée, l'éradication des foyers infectieux doit être rigoureuse et ne doit pas laisser de place au doute. C'est pourquoi l'attitude conservatrice, habituelle dans d'autre cas, doit être tempérée dans ces cas où le pronostic vital est en jeu.

\section{Bibliographie}

1. Le Moal G et al. Characteristics of brain abscess with isolation of anaerobic bacteria. Scand J Infect Dis 2003;35(5):318-21.

2. Rousse JM et al. Dialister pneumosintes associated with human brain abscesses. $\mathrm{J}$ Clin Microbiol 2002:40:3871-3.1994.

3. Brewer MS, MC Carty CS, Wellman WE.

Brain abcess: a review of recent experience. Ann Intern Med 1975; 82:571-6.

4. Ingham $H R$, Kalbag Danka RM, Tharagonnet D, High AS. Abscesses of the frontal lobe of the brain secondary to covert dental sepsis.
The Lancet 1978;vol 312. Issue 8088:497-499.

5. Greenlee JE. Anatomic considerations in central nervous system infections.

In: Mandell GL et al., ed. Principles and practice of infectious diseases. New York: Churchill Linvingstone 1995:821-831 (4e éd.).

6. Mathisen GE et al. Brain abscess.

Clin Infect 1997; Dis 25:763-781.

7. Larkin EB, Scott SD. Metastatic paraspinal abscess and paraplegia secundary to dental extraction. Br Dent J 1994;177:340-2.

8. Wispelwey B et al. Brain abscess in infections of the central nervous system.

In: Scheld WM et al., ed. Philadelphia: Lippincott-Raven 1997;463-491 (2e éd.).

9. Katlama C. SIDA et urgences. Rean Soins Intens Med Urg 1994;10:313-321.

10. Boisrame-Gastrin $S$, Legens $M$, Predine-Hug $F$. Abcès cérébraux d'origine dentaire, une porte d'entrée à ne pas méconnaître : à propos d'un cas. Médecine Buccale Chirurgie Buccale 2005;11(3):175-180.

11. Gendron $R$, Grenier $D$, Maheu-Mobert L. The oral cavity as a reservoir of bacterial pathogens for focal infections. Microb Infect 2000;2:897-906 


\section{Brain abscesses of dental origin: report of two cases}

Julien BALLY,

Rémi CURIEN,

Sophie BRENOT,

Alexandra SOURDOT,

Audrey $\mathrm{LE}$,

Pierre BRAVETTI
Even if the forecast of the brain abscesses has improved thanks to the antibiotherapy, imagery and surgery, their complications may often be lethal. Their etiologies are various; we report here two cases of brain abscesses of dental origin. In the light of these cases, it appears essential as in any focal infection to eradicate every dental focus.

\section{Keywords \\ - focal dental infections \\ - brain abscess}

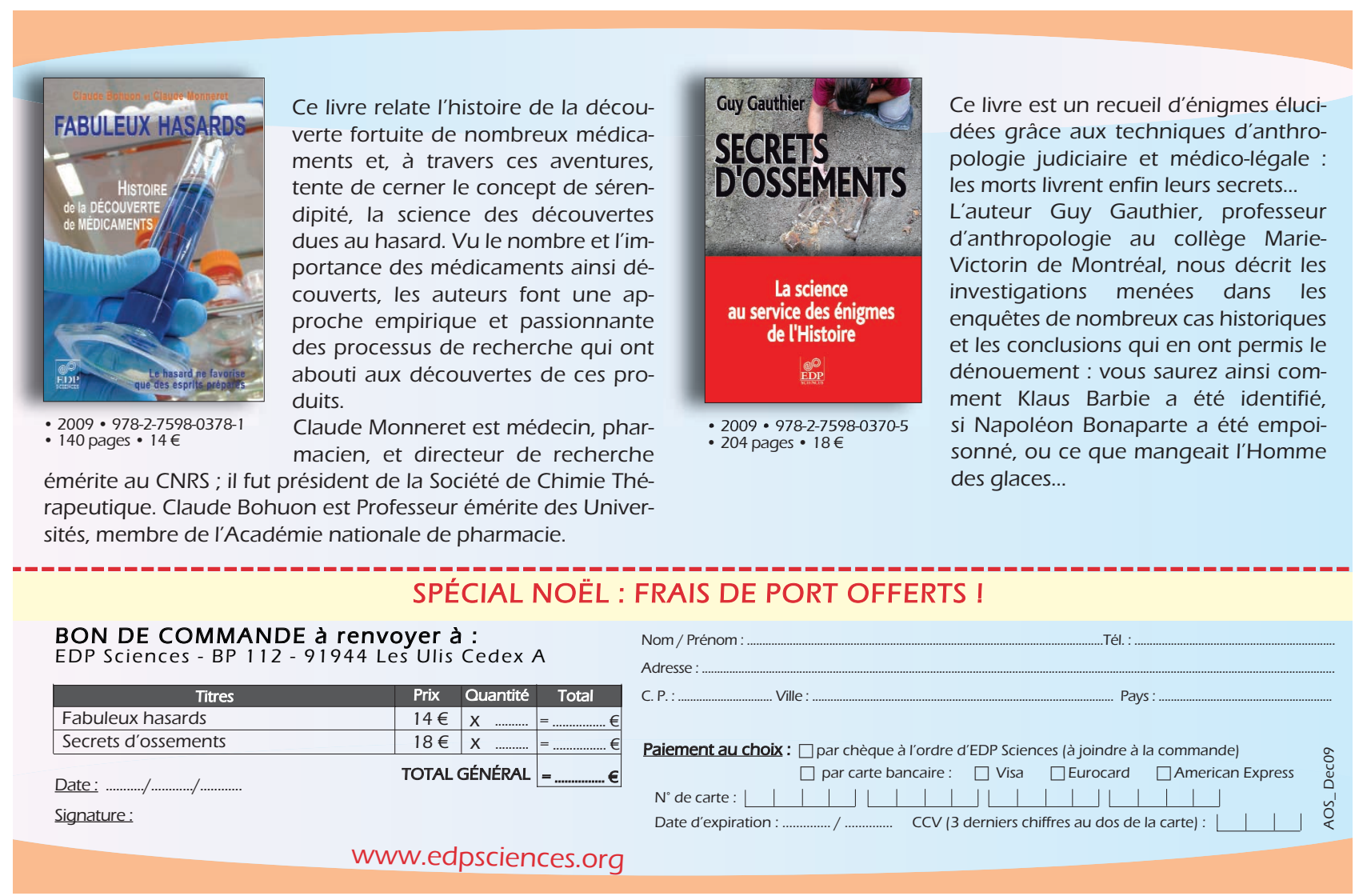

